\title{
Effect of Biodentine and Bioaggregate on odontoblastic differentiation via mitogen-activated protein kinase pathway in human dental pulp cells
}

\author{
J.-Y. Jung ${ }^{1,2}$, S.-M. Woo ${ }^{1,2}$, B.-N. Lee ${ }^{2,3}$, J.-T. Koh ${ }^{2}$, J. E. Nör ${ }^{4}$ \& Y.-C. Hwang ${ }^{2,3}$ \\ ${ }^{1}$ Department of Physiology, School of Dentistry, Dental Science Research Institute, Chonnam National University, Gwangju; \\ ${ }^{2}$ Research Center for Biomineralization Disorders. Chonnam National University, Gwangju; ${ }^{3}$ Department of Conservative \\ Dentistry School of Dentistry, Dental Science Research Institute, Chonnam National University, Gwangju, Korea; and \\ ${ }^{4}$ Angiogenesis Research Laboratory, Department of Cariology, Restorative Sciences, Endodontics, School of Dentistry, University \\ of Michigan, Ann Arbor, MI, USA
}

\begin{abstract}
Jung J-Y, Woo S-M, Lee B-N, Koh J-T, Nör JE, Hwang Y-C. Effect of Biodentine and Bioaggregate on odontoblastic differentiation via mitogen-activated protein kinase pathway in human dental pulp cells. International Endodontic Journal, 48, 177-184, 2015.
\end{abstract}

Aim To compare the mineralization inductive capacity of Biodentine and Bioaggregate with Mineral trioxide aggregate (MTA) and to investigate possible signaling pathways of mineralization in human dental pulp cells (HDPCs).

Methodology Viability of HDPCs in response to Biodentine, Bioaggregate, and MTA was measured using 3-[4,5-dimethylthiazol-2-yl]-2,5 diphenyltetrazolium bromide. To investigate their potential to induce odontoblast differentiation, expression of dentine sialophosphoprotein (DSPP) and dentine matrix protein1 (DMP1) mRNA level was evaluated by RTPCR. For the mineralized nodule assay, Alizarin red staining was performed. To determine the role of MAPK signaling in the odontoblastic differentiation of HDPCs, activated MAPKs were investigated by Western blot and the effect of MAPK inhibitor was examined by Alizarin red $\mathrm{S}$ staining. The results were

Correspondence: Yun-Chan Hwang, Department of Conservative Dentistry, School of Dentistry, Chonnam National University, Young-bong ro 77, Bukgu, Gwangju, Korea (Tel.: +82-62-530-5831; fax: +82-62-5305629; e-mail: ychwang@chonnam.ac.kr).

The first two authors J-Y Jung and S-M Woo contributed equally to this work as first authors. statistically analysed using one-way ANOVA and the Bonferroni test.

Results The effects of MTA, Biodentine, and Bioaggregate on cell viability were similar. Biodentine and Bioaggregate enhanced DSPP and DMP1 mRNA expression compared to the control group, but to the same extent as MTA $(P<0.05)$. MTA, Biodentine, and Bioaggregate increased the area of calcified nodules compared to the control $(P<0.01)$. MTA, Biodentine, and Bioaggregate increased phosphorylation of extracellular signal-regulated kinase (ERK), p38, and c-Jun N-terminal kinase (JNK). MAPK inhibitors attenuated mineralized nodule formation, which was increased by MTA, Biodentine, and Bioaggregate, respectively $(P<0.01)$.

Conclusion Biodentine and Bioaggregate stimulated odontoblastic differentiation and mineralization nodule formation by activating the MAPK pathway as did MTA. This suggests that the new materials could be useful for regenerative endodontic procedures.

Keywords: bioaggregate, biodentine, MAPK, mineralization, odontoblast.

Received 9 December 2013; accepted 14 April 2014

\section{Introduction}

Mineral trioxide aggregate (MTA) is used as a pulp capping agent on exposed pulp surfaces because it can induce formation of dentine bridges and tertiary dentine. During reparative dentinogenesis, odontoblast-like cells differentiate from dental pulp cells to pro- 
vide the replacement cells. MTA affects mineralization and differentiation of dental pulp cells (Yasuda et al. 2008, Masuda-Murakami et al. 2010) and the combination of MTA and enamel matrix derivative promotes odontoblastic differentiation of human dental pulp cells (HDPCs) (Min et al. 2009). Tricalcium silicate cement can also induce odontogenic differentiation of HDPCs and might be a candidate pulp capping agent (Peng et al. 2011).

Biodentine $^{\mathrm{TM}} \quad$ (Septodont, Saint-Maur-des-Fosses, France) has recently been developed and is marketed as a bioactive material. Biodentine induces differentiation of odontoblast-like cells and stimulates mineralization of murine pulp cells. Biodentine is considered a suitable pulp capping material (Zanini et al. 2012). Biodentine also increases expression of transforming growth factor-beta 1 (TGF- $\beta 1$ ) expression in human pulp cells and induces mineralization foci in a human tooth culture model (Laurent et al. 2012).

Bioaggregate (Innovative Bioceramix, Vancouver, $\mathrm{BC}$, Canada) has also been recently developed as a bioceramic material. Bioaggregate has cytotoxicity similar to MTA with human mesenchymal cells (De-Deus et al. 2009) and HDPCs (Chung et al. 2010). Bioaggregate induces mineralization associated gene expression in mouse osteoblast cell lines (Yuan et al. 2010). Bioaggregate is also similar to MTA in promoting odontoblastic differentiation of HDPCs (Chang et al. 2014).

Mitogen-activated protein kinases (MAPKs) are an essential component in many physiologic processes, such as cell growth, proliferation, differentiation, and apoptosis (Neary 1997). MAPKs are a family of serinethreonine kinases and include three familes, extracellular signal-regulated kinase (ERK), c-Jun N-terminal kinase (JNK), and p38 JNK (Johnson \& Lapadat 2002). p38 MAPK is activated during odontoblast stimulation in tertiary dentinogenesis (Simon et al. 2010).

Previous studies have demonstrated that the mineralization and odontoblastic differentiation effects of MTA can be explained by MAPK pathways and calcium ion release from MTA. MTA induces phosphorylation of MAPK, and both MAPK inhibitor and calcium channel blocker attenuated the MTA-induced odontoblastic differentiation (Zhao et al. 2012, Woo et al. 2013). However, the mechanism of mineralization effect of Biodentine and Bioaggregate is unclear. The purpose of this study was to compare the mineralization effect of Biodentine and Bioaggregate with MTA and reveal the possible pathway of mineralization in HDPCs.

\section{Material and methods}

\section{Preparation of MTA, Biodentine, and Bioaggregate, and material extract}

MTA, Biodentine, and Bioaggregate were mixed according to manufacturer's instructions under sterile conditions and then were added to moulds (diameter $5.0 \mathrm{~mm}$ and height $3.0 \mathrm{~mm}$ ). The materials were left to set for $4 \mathrm{~h}$ at $37{ }^{\circ} \mathrm{C}$ in a humidified $5 \% \mathrm{CO}_{2}$ incubator. Each disc was removed using sterile forceps. Discs of each material were placed into $50 \mathrm{~mL}$ of fresh alpha-minimal essential medium ( $\alpha$-MEM; Gibco Invitrogen, Grand Island, NY, USA) and incubated for 7 days at $37{ }^{\circ} \mathrm{C}$ in a humidified $5 \% \mathrm{CO}_{2}$ atmosphere. The medium was drawn off and sterilized by passage through a $0.2 \mu \mathrm{m}$ pore size filter before use. The concentrations of MTA, Biodentine, and Bioaggregate extracts were $0.1,1,10$, and $20 \mathrm{mg} / \mathrm{mL}$ for cell viability and $10 \mathrm{mg} \mathrm{mL}^{-1}$ for the other experiments.

\section{Cell culture}

HDPCs were isolated from freshly extracted maxillary third molars from three male patients. All procedures were conducted with informed consent. The institutional review board of the Chonnam National University Dental Hospital approved this study protocol (CNUDH-2013-002). Immediately after extraction, the teeth were split open and the pulp tissues aseptically removed and minced with a surgical knife. The minced tissues were placed in wells of 6-well cell culture plates containing $\alpha$-MEM supplemented with $10 \%$ fetal bovine serum (FBS), $100 \mathrm{U} \mathrm{mL}^{-1}$ penicillin, and $100 \mathrm{mg} \mathrm{mL}^{-1}$ streptomycin in a humidified atmosphere of $5 \% \mathrm{CO}_{2}$ at $37{ }^{\circ} \mathrm{C}$. After culturing for 14-20 days, outgrowth of the cells from pulp tissue was observed. When the cells reached confluence, subculture was performed. Cells passaged 3-4 times were used for this study.

\section{Cell viability assay}

Cell viability was measured using a 3-[4,5-dimethylthiazol-2-yl]-2,5 diphenyltetrazolium bromide MTT assay. Cells were seeded at a concentration of $2 \times 10^{4}$ cells/well in 96-well plates in $\alpha$-MEM with $10 \%$ FBS. The concentrations of MTA, Biodentine, and Bioaggregate extract were 0.1, 1, 10, and $20 \mathrm{mg} \mathrm{mL}^{-1}$, respectively, based on a previous report (Hakki et al. 2009). After incubation for 2 days, MTT 
was added to each well for the last 4 hs of the experiment, and the reaction was stopped by the addition of dimethylsulfoxide. The optical density was determined at $570 \mathrm{~nm}$ on a multiwell plate reader. The percentage of cell viability in the control group (media only group) represented $100 \%$.

\section{Reverse transcription-polymerase chain reaction}

HDPCs were incubated with $10 \mathrm{mg} \mathrm{mL}^{-1}$ of MTA, Biodentine, and Bioaggregate extract for 1 and 2 days. Total RNA of HDPCs was extracted using Trizol reagent (Life Technologies, Gaithersburg, MD, USA) according to the manufacturer's instructions. RNA $(2 \mu \mathrm{g})$ was reverse transcribed for first-strand complementary DNA (cDNA) synthesis (Gibco BRL, Rockville, MD, USA). The cDNA was amplified in a final volume of $20 \mu \mathrm{L}$ containing $2.5 \mathrm{mmol} / \mathrm{L}$ magnesium dichloride, 1.25 U Ex Taq polymerase (Bioneer, Daejeon, Korea), and $1 \mathrm{mmol} / \mathrm{L}$ specific primers. Amplification was performed for 30 cycles in a DNA thermal cycler. Primer sequences for differentiation markers are detailed in Table 1. The PCR products were resolved on a $1.5 \%$ agarose gel and stained with ethidium bromide. Gels were scanned and bands were quantified by optical densitometry.

\section{Alizarin red S staining}

For mineralized nodule assay, HDPCs were seeded in 24 well plates at a density of $5 \times 10^{4}$ cells/well and treated using $10 \mathrm{mg} \mathrm{mL}^{-1}$ of MTA, Biodentine, and Bioaggregate extract with or without pretreatment of MAPK inhibitors (U0126, $10 \mu \mathrm{mol} / \mathrm{L} ; \mathrm{SB} 203580$, $10 \mu \mathrm{mol} / \mathrm{L}$; and SP600125, $10 \mu \mathrm{mol} / \mathrm{L})$ for $1 \mathrm{~h}$. The culture medium was replaced with fresh culture medium every 2 days. After 14 days, the calcium deposition of HDPCs was studied using $0.1 \%$ Alizarin red S staining solution (Sigma-Aldrich, St. Louis, MO, USA). The samples were washed with phosphate buffered

Table 1 RT-PCR primer sequence

\begin{tabular}{llc}
\hline Gene & \multicolumn{1}{c}{ Sequence $\left(5^{\prime}-3^{\prime}\right)$} & Size $(\mathrm{bp})$ \\
\hline DSPP & Forward: CAGTGATGAATCTAATGG & 488 \\
& Reverse: CTGATTTGCTGCTGTCTGAC & \\
DMP1 & $\begin{array}{l}\text { Forward: CAGGAGCACAGGAAAAGGAG } \\
\text { Reverse: CTGGTGGTATCTTGGGCACT }\end{array}$ & 213 \\
GAPDH & $\begin{array}{l}\text { Forward: GAGTCAACGGATTTGGTCGT } \\
\text { Reverse: GACAAGCTTCCCGTTCTCAG }\end{array}$ & 185 \\
\hline
\end{tabular}

DSPP, dentine sialophosphoprotein; DMP1, dentine matrix protein1; GAPDH, glyceraldehyde-3-phosphate dehydrogenase. saline (PBS) and fixed with 70\% ice-cold ethanol for $1 \mathrm{~h}$ at room temperature, rinsed twice with distilled water, and stained with $40 \mathrm{mmol} / \mathrm{L}$ Alizarin red S ( $\mathrm{pH} 4.2$ ) solution for $15 \mathrm{~min}$ with gentle agitation at room temperature. Alizarin red S staining was photographed using an Officejet pro L7580 scanner (HP, Palo Alto, CA, USA). Bound dye was solubilized in $10 \%$ cetylpyridinium chloride and quantitated spectrophotometrically at $562 \mathrm{~nm}$.

\section{Western blot analysis}

HDPCs were incubated with $10 \mathrm{mg} \mathrm{mL}^{-1}$ of MTA, Biodentine, and Bioaggregate extract for 15, 30, 60, and $120 \mathrm{~min}$. Cell lysates $(50 \mu \mathrm{g})$ were placed in NP-40 lysis buffer $\left(30 \mathrm{~m} \mathrm{~mol} \mathrm{~L}^{-1}\right.$ Tris-Cl, pH 7.5, $1 \mathrm{mmol} \mathrm{L}^{-1}$ EDTA, $150 \mathrm{mmol} \mathrm{L}{ }^{-1} \mathrm{NaCl}, 1 \% \mathrm{NP}-40$, $1 \mathrm{mmol} \mathrm{L}^{-1}$ phenylmethylsufonyl fluoride, and protease inhibitor mixture containing $1 \mu \mathrm{g} \mathrm{mL}^{-1}$ aprotinin and leupeptin) and separated by $12 \%$ sodium dodecyl sulfate-polyacrylamide gel electrophoresis. The resolved proteins were transferred to a nitrocellulose membrane (Amersham Pharmacia Biotech, Little Chalfont, UK) according to standard procedures. The membrane was blocked in 5\% non-fat dry milk for $1 \mathrm{~h}$ and incubated with anti-phosphoERK, anti-ERK, anti-phospho-p38, anti-p38, antiphospho-JNK, or anti-JNK antibody (Cell Signaling Technology, Danvers, MA, USA) overnight at $4{ }^{\circ} \mathrm{C}$. After incubation with the specific peroxidase-coupled secondary antibody (Sigma-Aldrich) for $1 \mathrm{~h}$, the blotted bands were detected using an enhanced chemiluminescence detection kit (Amersham Pharmacia Biotech).

\section{Statistical analysis}

All experiments were performed in triplicate. The data are expressed as the mean \pm standard deviation and were analysed using a one-way analysis of variance followed by the Bonferroni post hoc test with the use of the SPSS 17.0 software program (SPSS, Chicago, IL, USA).

\section{Results}

\section{Effect of Biodentine and Bioaggregate on cell viability in HDPCs}

To determine the optimal concentration of MTA, Biodentine, and Bioaggregate, viability of HDPCs was 


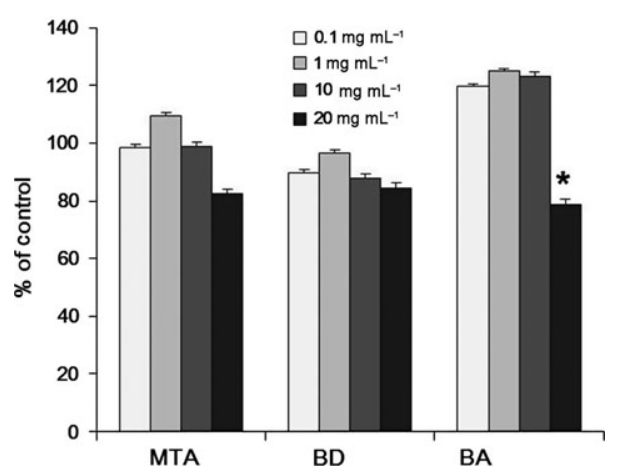

Figure 1 Effects of MTA, Biodentine, and Bioaggregate on HDPC viability. The cell viability in the control group (media only group) represented $100 \%$ as the reference value. The asterisk $(*)$ indicates a significant difference when compared to other concentrations $(P<0.05)$. Each value represents the mean of three replicates.

evaluated. Overall, MTA, Biodentine, and Bioaggregate similarly affected HDPC viability. However, $20 \mathrm{mg} \mathrm{mL} \mathrm{m}^{-1}$ of Bioaggregate produced lower cell viability than other concentrations (Fig. 1). Therefore, $10 \mathrm{mg} \mathrm{mL}^{-1}$ was used in subsequent experiments.

Effect of Biodentine and Bioaggregate on the expression of DSPP and DMP1 mRNA in HDPCs

To investigate the effect of Biodentine and Bioaggregate on odontoblast differentiation in HDPCs, expressions of dentine sialophosphoprotein (DSPP) and dentine matrix protein1 (DMP1) mRNA were evaluated by RT-PCR and quantified with densitometry. Biodentine and Bioaggregate increased DSPP and DMP1 mRNA expression compared to the untreated control at day 1. MTA, Biodentine, and Bioaggregate increased DMP1 mRNA expression and Biodentine increased DSPP mRNA expression compared to the untreated control at day 2 (Fig. 2) $(P<0.05)$.

Biodentine and Bioaggregate induce mineralization nodules in HDPCs

To determine the mineralization effect of Biodentine and Bioaggregate in HDPCs, mineralized nodule formation in HDPCs was examined using Alizarin Red S staining. After 14 days of culture, MTA, Biodentine, and Bioaggregate enhanced the area of calcified nodules compared to the untreated control (Fig. 3) $(P<0.01)$.
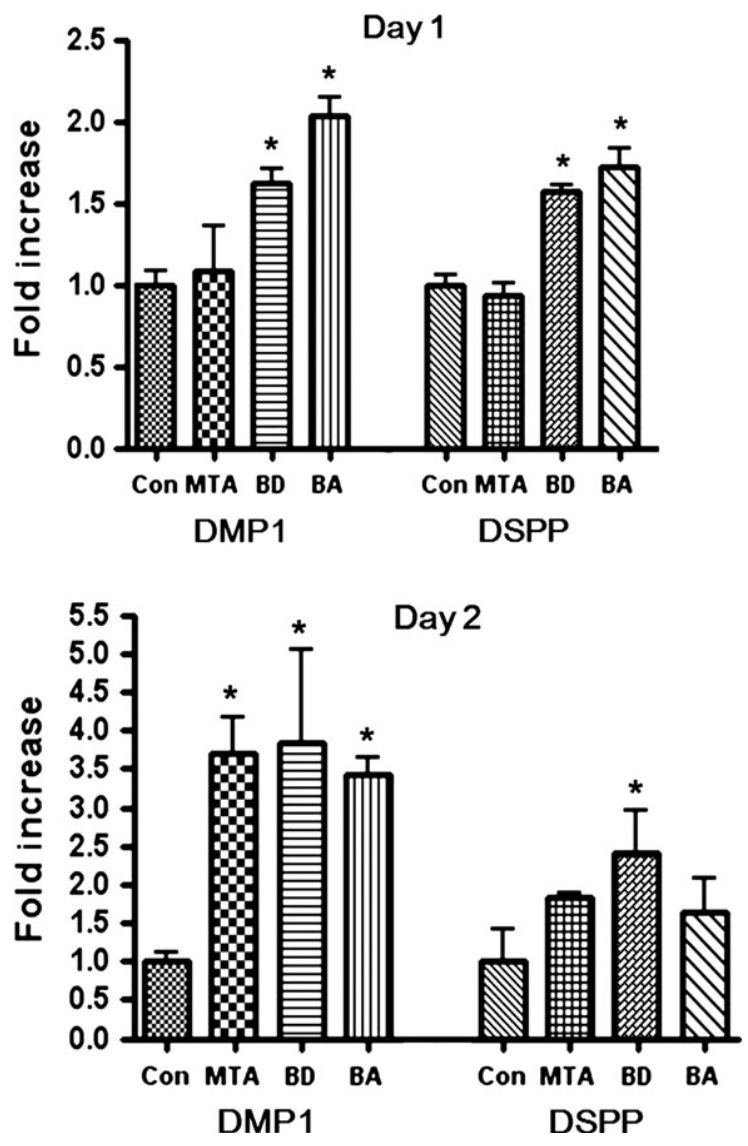

Figure 2 Effects of MTA, Biodentine, and Bioaggregate on HDPCs differentiation. he mRNA levels of DSPP and DMP1 were determined by RT-PCR and quantified by densitometry. Values are expressed as mean \pm standard deviation of three separate experiments. *Statistically significant difference when compared to control $(P<0.05)$.

\section{Biodentine and Bioaggregate induce MAPK activation in HDPCs}

To investigate the activation of MAPK signaling in MTA-, Biodentine -, and Bioaggregate -induced odontoblastic differentiation in HDPCs, phosphorylated MAPK was investigated by Western blot. MTA, Biodentine, and Bioaggregate induced phosphorylation of ERK, p38, and JNK within $30 \mathrm{~min}$ of treatment (Fig. 4). To determinate the role of MAPK signaling on odontoblastic differentiation in HDPCs, cells were pretreated with $10 \mu \mathrm{mol} \mathrm{L}^{-1}$ of MAPK inhibitors (U0126, SB203580, and SP600125) for $1 \mathrm{~h}$, cultured with $10 \mathrm{mg} \mathrm{mL}^{-1} \mathrm{MTA}$, Biodentine, and Bioaggregate extract for 14 days, and stained using Alizarin red. Inhibited activation of ERK, p38, and JNK in 
(a) 7 days

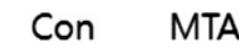

$B D$

BA

14 days
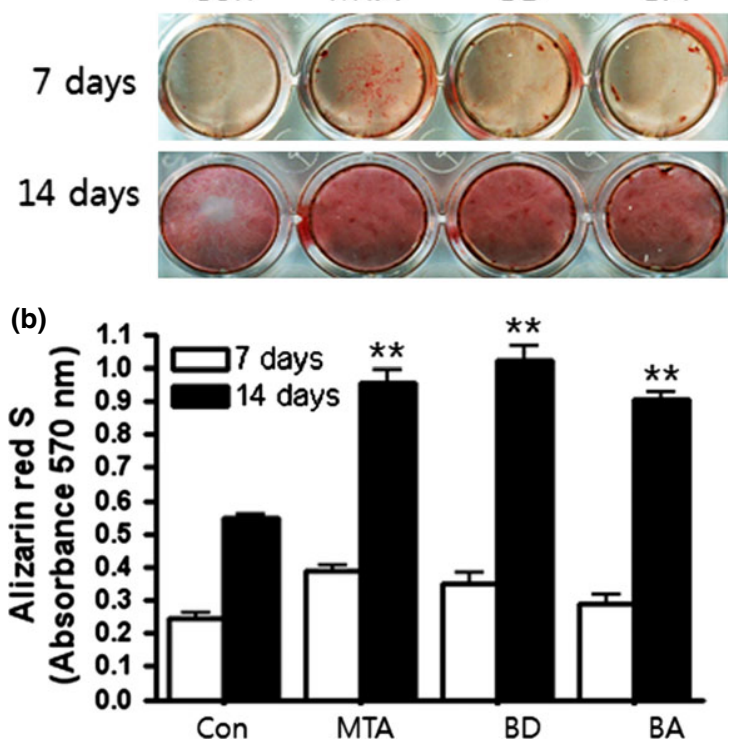

Figure 3 Effects of MTA, Biodentine, and Bioaggregate on mineralization of HDPCs. (a) Representative photograph of calcified nodule. (b) The quantification of Alizarin red S staining. The results are representative of three independent experiments. **Statistically significant difference when compared to control $(P<0.01)$.

MTA, Biodentine, and Bioaggregate extract-treated HDPCs with U0126, SB203580, and SP600125, respectively, reduced mineralization nodule formation, which was increased by MTA, Biodentine, and Bioaggregate extract (Fig. 5) $(P<0.01)$.

\section{Discussion}

MTA stimulates the odontogenic differentiation of HDPCs and the effects are mediated by the ERK pathway (Simon et al. 2010, Zhao et al. 2012, Woo et al.
2013). Biodentine or Bioaggregate also stimulate odontoblastic differentiation of HDPCs (Laurent et al. 2012, Chang et al. 2014). The present study focused on whether the effects of Biodentine and Bioaggregate are comparable to MTA in odontoblastic differentiation and mineralization, and whether MAPK pathways are involved in this process.

The evaluation of cytotoxicity is crucial when treating with Biodentine and Bioaggregate because they directly contact the pulpal tissues. Cell viability assay was performed to determine the difference in cell damage. In this study, Biodentine and Bioaggregate showed similar viability in HDPCs at $10 \mathrm{mg} \mathrm{mL}^{-1}$ compared with MTA-treated group.

The mineralization effect of the pulp capping material is very important in clinical situations. MTA has mineralization effects that include odontogenic or cementogenic activities (Tani-Ishii et al. 2007, Yasuda et al. 2008, Hakki et al. 2009). DSPP has a regulatory role in the mineralization of reparative dentine and is regarded as a specific marker of odontoblast differentiation (Liu et al. 2013). DMP1 is present in the extracellular matrix of dentine and bone, and serves as an essential factor for mineralization of hard tissue (Suzuki et al. 2012). In addition, calcium nodule production is a marker of mineralization (Chang et al. 2014). In this study, to determine the odontoblastic differentiation and mineralization effect of the Biodentine and Bioaggregate, odontoblastic differentiation marker genes, such as DMP1 and DSPP, were evaluated by RT-PCR and mineralized nodule formation was examined using Alizarin red S staining during HDPC culture with Biodentine and Bioaggregate extract. Biodentine and Bioaggregate up-regulated DSPP and DMP-1 mRNA. Interestingly, Biodentine and Bioaggregate showed higher expression than the MTA-treated group at

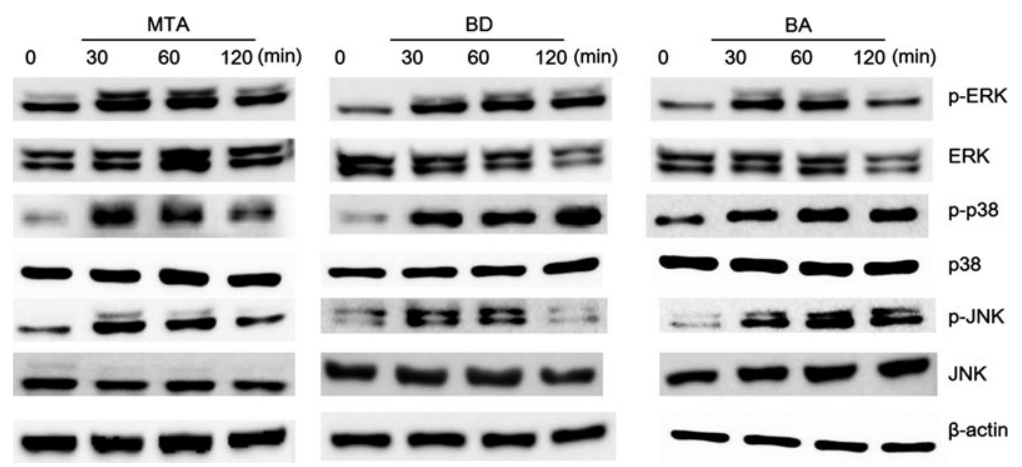

Figure 4 Effects of MTA, Biodentine, and Bioaggregate on MAPK phosphorylation in HDPCs. Phosphorylation of MAPK was detected by Western blot. The expression of $\beta$-actin was used as the control. 
(a)
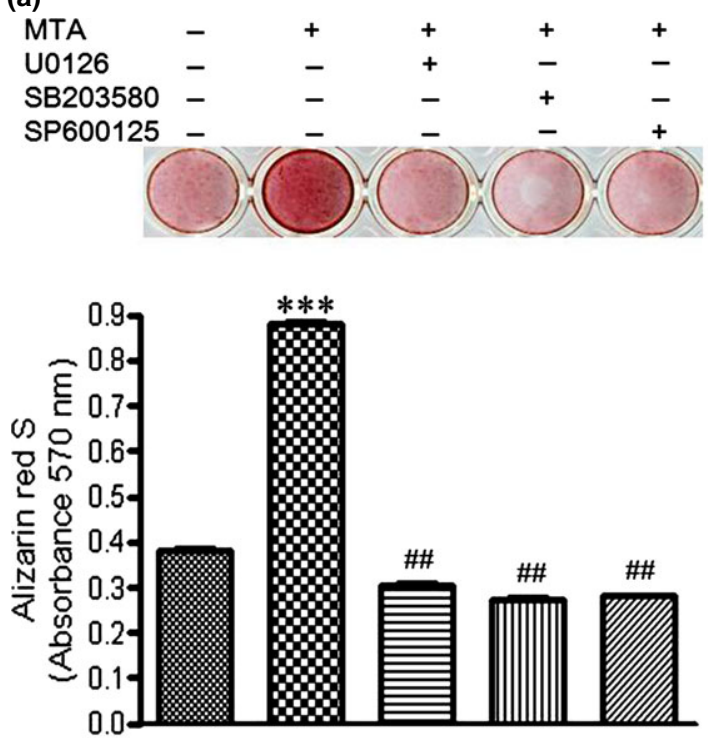

(b)
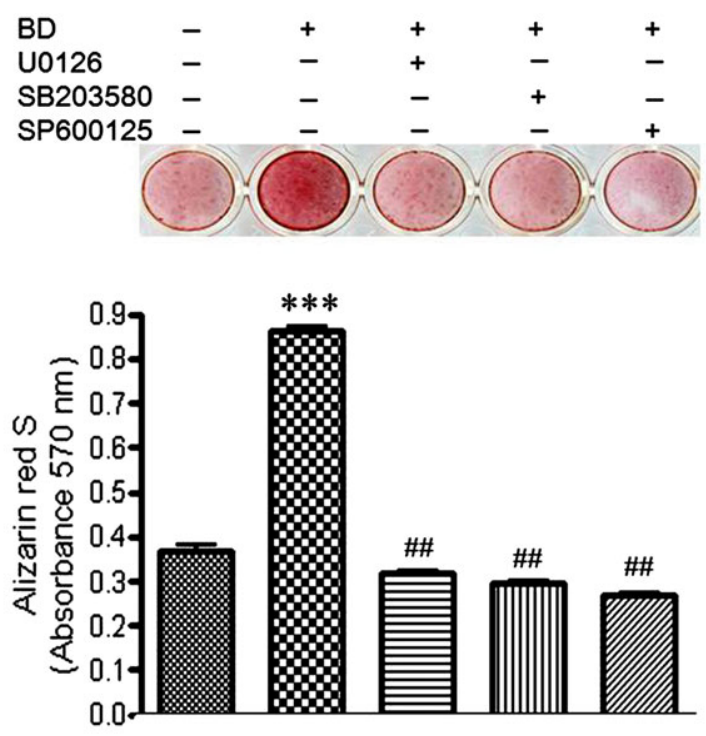

(c)
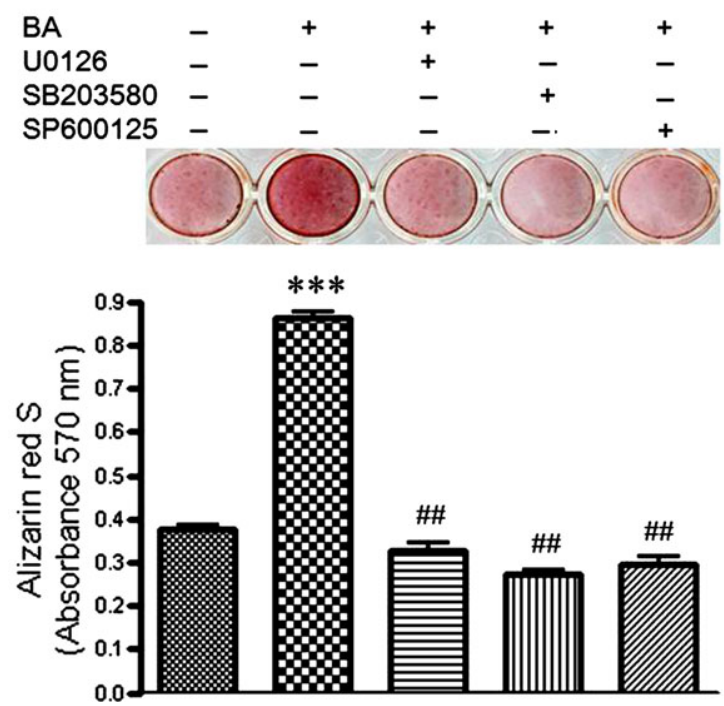

Figure 5 Involvement of MAPK activation in MTA-, Biodentine-, and Bioaggregate -induced mineralization in HDPCs. U0126, SB203580, and SP600125 reduced mineralizaed nodule formation which was increased by (a) MTA, (b) Biodentine, and (c) Bioaggregate. The results are representative of three independent experiments. ***Statistically significant difference when compared to control $(P<0.001)$. \#\#Statistically significant difference when compared to MTA-, Biodentine-, and Bioaggregatetreated group $(P<0.01)$.

1 day. In addition, Alizarin red S staining revealed that Biodentine and Bioaggregate induced similar levels of mineralization nodule formation compared with MTA-treated group after 14 days of HDPC culture. These results are consistent with the previous findings that Bioaggregate increases DSPP and DMP1 mRNA expression and mineralization nodule formation (Chang et al. 2014), and that Biodentine increases calcific nodule formation in immortalized murine pulp cells (Zanini et al. 2012).

Considering the present results, it seems that the clinical application of Biodentine and Bioaggregate as pulp capping agents promotes mineralization of dentine beneath the capping material, and then stimulates reparative odontogenesis from injured dental pulp tissue. Additional studies will be necessary to 
compare cytokine production or inflammatory response with these cements and MTA in HDPCs for clinical application.

The mechanism by which MTA induces odontoblastic differentiation in HDPCs is not completely understood. MTA induces mineralization and differentiation by MAPK pathway and calcium ion release in HDPCs (Simon et al. 2010, Zhao et al. 2012, Woo et al. 2013). MTA also activates ERK, p38, and JNK MAPKs during odontoblast differentiation and MAPK pathways, particularly ERK, are involved in the MTAinduced odontoblast differentiation of HDPCs (Zhao et al. 2012). On the other hand, it is reported that p38 MAPK is activated during odontoblast stimulation in tertiary dentinogenesis (Simon et al. 2010). In addition, influx of calcium ion released from MTA stimulates odontoblastic differentiation and mineralization via MAPK pathway activation (Woo et al. 2013).

In this study, MAPK pathways were associated with Biodentine - and Bioaggregate -induced mineralization in HDPCs was investigated. Biodentine and Bioaggregate induced phosphorylation of ERK, p38, and JNK MAPKs. These results are consistent with previous results for MTA (Zhao et al. 2012). MAPK inhibitors (U0126, SB203580, and SP600125) abolished the mineralization nodule formation, which was increased by Biodentine and Bioaggregate extracts. These results indicated that ERK, p38, and JNK MAPK pathways play a crucial role in Biodentine and Bioaggregate -induced mineralization in HDPCs.

\section{Conclusion}

Collectively, these data demonstrate that Biodentine and Bioaggregate induce mineralization of HDPCs by activating the MAPK pathway. These results are comparable to those observed with HDPCs exposed to the gold standard, MTA, suggesting the value of Bioaggregate and Biodentine for regenerative endodontic procedures.

\section{Acknowledgements}

The authors deny any conflicts of interest related to this study.

This study was supported by the Basic Science Research Program through the National Research Foundation of Korea(NRF) funded by the Ministry of
Education, Science and Technology (2011-0029663 and 2012R1A1A2005002)) and the National Research Foundation of Korea(NRF) grant funded by the Korea government(MEST) (No. 2011-0030121).

\section{References}

Chang SW, Lee SY, Kum KY, Kim EC (2014) Effects of ProRoot MTA, Bioaggregate, and Micromega MTA on odontoblast differentiation of human dental pulp cells. Journal of Endodontics 40, 113-8.

Chung CR, Kim ES, Shin SJ (2010) Biocompatibility of Bioaggregate cement on human pulp and periodontal ligament (PDL) derived cells. Restorative Dentistry \& Endodontics 35 , 473-8.

De-Deus G, Canabarro A, Alves G, Linhares A, Senne MI, Granjeiro JM (2009) Optimal cytocompatibility of a bioceramic nanoparticulate cement in primary human mesenchymal cells. Journal of Endodontics 35, 1387-90.

Hakki SS, Bozkurt SB, Hakki EE, Belli S (2009) Effects of mineral trioxide aggregate on cell survival, gene expression associated with mineralized tissues, and biomineralization of cementoblasts. Journal of Endodontics 35, 513-9.

Johnson GL, Lapadat R (2002) Mitogen-activated protein kinase pathways mediated by ERK, JNK, and p38 protein kinases. Science 298, 1911-2.

Laurent P, Camps J, About I (2012) Biodentine(TM) induces TGF-beta1 release from human pulp cells and early dental pulp mineralization. International Endodontic Journal 45, 439-48.

Liu H, Lin H, Zhang L et al. (2013) miR-145 and miR-143 regulate odontoblast differentiation through targeting Klf4 and Osx genes in a feedback loop. Journal of Biological Chemistry 288, 9261-71.

Masuda-Murakami Y, Kobayashi M, Wang X et al. (2010) Effects of mineral trioxide aggregate on the differentiation of rat dental pulp cells. Acta Histochemica 112, 452-8.

Min KS, Yang SH, Kim EC (2009) The combined effect of mineral trioxide aggregate and enamel matrix derivative on odontoblastic differentiation in human dental pulp cells. Journal of Endodontics 35, 847-51.

Neary JT (1997) MAPK cascades in cell growth and death. Physiology 12, 286-93.

Peng W, Liu W, Zhai W et al. (2011) Effect of tricalcium silicate on the proliferation and odontogenic differentiation of human dental pulp cells. Journal of Endodontics 37, 1240-6.

Simon S, Smith AJ, Berdal A, Lumley PJ, Cooper PR (2010) The MAP kinase pathway is involved in odontoblast stimulation via p38 phosphorylation. Journal of Endodontics 36, $256-9$.

Suzuki S, Haruyama N, Nishimura F, Kulkarni AB (2012) Dentin sialophosphoprotein and dentin matrix protein-1: two highly phosphorylated proteins in mineralized tissues. Archive of Oral Biology 57, 1165-75. 
Tani-Ishii N, Hamada N, Watanabe K, Tujimoto Y, Teranaka T, Umemoto T (2007) Expression of bone extracellular matrix proteins on osteoblast cells in the presence of mineral trioxide. Journal of Endodontics 33, 836-9.

Woo SM, Hwang YC, Lim HS et al. (2013) Effect of nifedipine on the differentiation of human dental pulp cells cultured with mineral trioxide aggregate. Journal of Endodontics 39 , 801-5.

Yasuda Y, Ogawa M, Arakawa T, Kadowaki T, Saito T (2008) The effect of mineral trioxide aggregate on the mineralization ability of rat dental pulp cells: an in vitro study. Journal of Endodontics 34, 1057-60.
Yuan Z, Peng B, Jiang H, Bian Z, Yan P (2010) Effect of Bioaggregate on mineral-associated gene expression in osteoblast cells. Journal of Endodontics 36, 1145-8.

Zanini M, Sautier JM, Berdal A, Simon S (2012) Biodentine induces immortalized murine pulp cell differentiation into odontoblast-like cells and stimulates biomineralization. Journal of Endodontics 38, 1220-6.

Zhao X, He W, Song Z, Tong Z, Li S, Ni L (2012) Mineral trioxide aggregate promotes odontoblastic differentiation via mitogen-activated protein kinase pathway in human dental pulp stem cells. Molecular Biology Reports 39 , 215-20. 